\title{
Risk of early progression according to circulating ESR1 mutation, CA-15.3 and cfDNA increases under first-line anti- aromatase treatment in metastatic breast cancer
}

Florian Clatot ${ }^{1,2^{*}}$ (D), Anne Perdrix ${ }^{2,3}$, Ludivine Beaussire ${ }^{2}$, Justine Lequesne ${ }^{4}$, Christelle Lévy ${ }^{5}$, George Emile ${ }^{5}$, Michael Bubenheim ${ }^{6}$, Sigrid Lacaille ${ }^{2}$, Céline Calbrix ${ }^{3}$, Laetitia Augusto ${ }^{1}$, Cécile Guillemet ${ }^{1}$, Cristina Alexandru ${ }^{1}$, Maxime Fontanilles ${ }^{1,2}$, David Sefriouil ${ }^{2}$, Lucie Burel ${ }^{4}$, Sabine Guénot ${ }^{4}$, Doriane Richard ${ }^{4}$, Nasrin Sarafan-Vasseur ${ }^{2}$ and Frédéric Di Fiore ${ }^{1,2}$

\begin{abstract}
Background: Endocrine therapy is recommended as a first-line treatment for hormone receptor-positive metastatic breast cancer $(\mathrm{HR}+\mathrm{MBC})$ patients. No biomarker has been validated to predict tumor progression in that setting. We aimed to prospectively compare the risk of early progression according to circulating ESR1 mutations, CA-15.3, and circulating cell-free DNA in MBC patients treated with a first-line aromatase inhibitor (Al).

Methods: Patients with MBC treated with a first-line Al were prospectively included. Circulating biomarker assessment was performed every 3 months. The primary objective was to determine the risk of progression or death at the next follow-up visit (after 3 months) in case of circulating ESR1 mutation detection among patients treated with a first-line Al for HR+MBC.

Results: Overall, 103 patients were included, and 70 (68\%) had progressive disease (PD). Circulating ESR1 mutations were detected in 22/70 patients with PD and in 0/33 patients without progression $(p<0.001)$. Among the ESR1mutated patients, 18/22 had a detectable mutation prior to progression, with a median delay of 110 days from first detection to PD. The detection of circulating ESR1 mutations was associated with a 4.9-fold ( $95 \% \mathrm{Cl} 3.0-8.0)$ increase in the risk of PD at 3 months. Using a threshold value of $25 \%$ or $100 \%$, a CA-15.3 increase was also correlated with progression ( $p<0.001$ and $p=0.003$, respectively). In contrast to ESR1, the CA-15.3 increase occurred concomitantly with PD in most cases, in $27 / 47$ (57\%) with a 25\% threshold and in 21/25 (84\%) with a $100 \%$ threshold. Using a threshold value of either $25 \%$ or $100 \%$, cfDNA increase was not correlated with progression. (Continued on next page)
\end{abstract}

\footnotetext{
* Correspondence: florian.clatot@chb.unicancer.fr

'Department of Medical Oncology, Centre Henri Becquerel, Rouen, France ${ }^{2}$ Normandie Univ, UNIROUEN, Inserm U1245, IRON group, Rouen University Hospital, Normandy Centre for Genomic and Personalized Medicine, Rouen, France

Full list of author information is available at the end of the article
}

(c) The Author(s). 2020 Open Access This article is licensed under a Creative Commons Attribution 4.0 International License, which permits use, sharing, adaptation, distribution and reproduction in any medium or format, as long as you give appropriate credit to the original author(s) and the source, provide a link to the Creative Commons licence, and indicate if changes were made. The images or other third party material in this article are included in the article's Creative Commons licence, unless indicated otherwise in a credit line to the material. If material is not included in the article's Creative Commons licence and your intended use is not permitted by statutory regulation or exceeds the permitted use, you will need to obtain permission directly from the copyright holder. To view a copy of this licence, visit http://creativecommons.org/licenses/by/4.0/. The Creative Commons Public Domain Dedication waiver (http://creativecommons.org/publicdomain/zero/1.0/) applies to the data made available in this article, unless otherwise stated in a credit line to the data. 
(Continued from previous page)

Conclusion: The emergence of circulating ESR1 mutations is associated with a 4.9-fold increase in the risk of early $\mathrm{PD}$ during $\mathrm{Al}$ treatment in $\mathrm{HR}+\mathrm{MBC}$. Our results also highlighted that tracking circulating ESR1 mutations is more relevant than tracking CA-15.3 or cfDNA increase to predict progression in this setting.

Trial registration: ClinicalTrials.gov, NCT02473120. Registered 16 June 2015-retrospectively registered after one inclusion (first inclusion 1 June 2015)

Keywords: ESR1 mutation, Breast cancer, Circulating DNA, CA-15.3, Cell-free DNA, Aromatase inhibitor

\section{Introduction}

Tumor monitoring under treatment is currently based on clinical evaluation and imaging. In this context, the identification of early markers correlated with response to treatment is warranted to make real-time therapeutic adaptations. In metastatic breast cancer $(\mathrm{MBC})$, an abnormal level or an elevation of CA-15.3 contributes to identifying tumor progression in conjunction with imaging, history of disease, and clinical course [1]. Until now, evidence has been too low to recommend the use of CA-15.3 instead of conventional follow-up.

In this context, liquid biopsy offers new perspectives for the real-time monitoring of tumor response under treatment. It has been shown that circulating tumor cells (CTCs) can be isolated in plasma, with a high concentration of CTCs correlated with poor prognosis [2]. Moreover, cell-free DNA (cfDNA) or circulating tumor DNA (ctDNA) has also been intensively evaluated in that setting. cfDNA is related to cell turnover, combining both normal DNA and tumoral DNA. Among cancer patients, the cfDNA level is correlated with disease stage [3] and is easily identifiable and quantifiable within all patients. Few data are available regarding the prognostic value of cfDNA in MBC. In a prospective study on 268 patients treated for MBC with first-line chemotherapy, cfDNA concentration was reported as an independent prognostic factor for both overall survival (OS) and progression-free survival (PFS) [4]. On the other hand, ctDNA detection, which is based on the determination of a circulating molecular alteration specific to tumoral DNA, has also shown promising results for disease monitoring.

To date, the first-line treatment of patients with hormonal receptor-positive $\mathrm{MBC}(\mathrm{HR}+\mathrm{MBC})$ is based on aromatase inhibitors (AIs) combined with cdk4/6 inhibitors [5]. There are multiple mechanisms leading to resistance to endocrine therapy. Among them, mutations of the estrogen receptor gene (ESR1) have been associated with acquired resistance to AIs, with or without combination with cdk $4 / 6$ inhibitors $[6,7]$. The detection of these mutations at progression with AIs is observed in $30-50 \%$ of cases and is associated with a poor outcome $[6,8]$. Interestingly, these mutations can reliably be detected in blood, either by digital droplet PCR (ddPCR) [9-12] or by nextgeneration sequencing $[12,13]$. Furthermore, the detection of circulating ESR1 mutations several months before clinical progression has been observed in most patients $[8,14]$. These criteria make ESR1 mutations a potential biomarker for biological follow-up and therapeutic adaptation during AI treatment in advanced breast cancer.

To our knowledge, only one prospective study compared serial circulating biomarkers namely CTCs, ctDNA, and CA-15.3 in MBC [15]. Among 52 patients, $30(58 \%)$ were finally analyzed for ctDNA mainly based on PIK3CA and TP53 mutations. In these patients, ctDNA detection was found in at least one of the samples in 29 of the 30 patients (97\%), and CA-15.3 was elevated in at least one of the samples in 21 of the 27 patients (78\%). ctDNA had a better sensitivity and stronger association with tumor burden than CA-15.3 and CTCs. Moreover, while the emergence of circulating ESR1 mutations has been identified as a predictive marker of AI resistance in $\mathrm{HR}+\mathrm{MBC}$, data comparing the effectiveness of ctDNA versus CA-15.3 or cfDNA in that setting are lacking. In this context, we aimed to assess CA-15.3, cfDNA, and circulating ESR1 mutations to determine early progression in a prospective cohort of $\mathrm{HR}+\mathrm{MBC}$ patients treated with a first-line AI.

\section{Patients and methods Study design}

This study is based on an observational prospective cohort including $\mathrm{HR}+\mathrm{MBC}$ patients treated with a firstline AI for MBC from June 2015. Due to the evolution of the knowledge regarding circulating ESR1 mutations in 2016, an amendment regarding the objectives was accepted by regulatory agencies in January 2017, before the end of the inclusions and before any analysis. The inclusion criteria were as follows: women $\geq 18$ years with $\mathrm{MBC}$ or non-operable locally advanced $\mathrm{BC}$ and treatment with $\mathrm{AI}$ initiated at inclusion or at least 6 months before with a documented non-progressive disease. Previous treatment for early $\mathrm{BC}$ with chemotherapy/tamoxifen/fulvestrant or AI was allowed with a time frame of 2 years between the last treatment and metastatic evolution. The exclusion criteria were participation in another 
clinical trial and hormone receptor-negative $\mathrm{BC}$. The study was performed in the Henri Becquerel Cancer Centre, Rouen, France, and in the François Baclesse Cancer Centre, Caen, France. All patients provided informed consent, and the study was approved by an independent ethics committee. This prospective cohort was registered at www.clinicaltrials.gov (NCT02473120).

All included patients were followed up every 3 months with clinical examination and CT scan. Blood samples for circulating marker analysis were collected every 3 months using 2 tubes of $5 \mathrm{~mL}$ for ctDNA and cfDNA and using one tube of $5 \mathrm{~mL}$ for CA-15.3. A progressive disease (PD) was determined using the radiological evaluation by RECIST 1.1 [16] and the physician clinical evaluation. Each PD was confirmed by the Metastatic Breast Board of each center. Overall survival (OS) and progression-free survival (PFS) were defined as the time from AI initiation to death and the time from AI initiation to progression or death, respectively, and were censored at the last follow-up. Of note, OS and PFS at progression on $\mathrm{AI}$ were defined as the time from $\mathrm{PD}$ on AI to death and the time from PD on AI to progression or death, respectively. All patients with HER2-positive tumors were treated with anti-HER2 therapy.

\section{Plasma DNA extraction}

Blood samples were collected in EDTA tubes and processed within $3 \mathrm{~h}$ after collection. First, the tubes were centrifuged at $1000 \mathrm{~g}$ for $10 \mathrm{~min}$ at $4{ }^{\circ} \mathrm{C}$. Then, plasma was transferred to micro-tubes and centrifuged at $16000 \mathrm{~g}$ for $10 \mathrm{~min}$ at $4^{\circ} \mathrm{C}$. The plasma was then transferred to cryovials and stored at $-20^{\circ} \mathrm{C}$ for $24 \mathrm{~h}$, then at $-80^{\circ} \mathrm{C}$ until analysis. DNA was extracted from 2 to 3 $\mathrm{mL}$ of plasma using the QIAamp ${ }^{\circ}$ Circulating Nucleic Acid Kit (Qiagen, Hilden, Germany). Double-stranded DNA quantification was performed by a fluorometric method using the Quant-iT PicoGreen dsDNA Assay Kit (Invitrogen, Carlsbad, CA, USA) and a Twinkle LB970 microplate fluorometer (Berthold, BadWildbad, Germany).

\section{Circulating analyses}

The CA-15.3 assay was performed by the BRAHMS Kryptor Plus compact controller using TRACE (TimeResolved Amplified Cryptate Emission) technology. CA15.3 was considered elevated when it was above the normal upper limit $(30 \mathrm{U} / \mathrm{mL})$. We considered a CA-15.3 increase as the first occurrence of a CA-15.3 increase of either $25 \%$ or $100 \%$, between the CA- 15.3 nadir and every 3 months of follow-up. Similar variation rates (25 and $100 \%$ ) were also used for cfDNA analyses.

A droplet-based dPCR (ddPCR) platform (Qx200 ddPCR System, Bio-Rad Laboratories, Hercules, CA, USA) was used for the detection of mutant circulating
DNA in plasma samples. The positive samples were discriminated from the negative samples by using two multiplex ESR1 assays (dHsaMDXE91450042 ESR1 Multiplex 1 targeting E380Q, D538G, Y537C, and L536R mutations and dHsaMDXE65719815 ESR1 Multiplex 2 targeting S463P, Y537S, and Y537N mutations) (Bio-Rad Laboratories). The ddPCR reactions were performed in triplicate with $8 \mu \mathrm{L}$ or $40 \mathrm{ng}$ (depending on the amount) of cfDNA. A result was considered positive when 2 out of the 3 wells were positive. In the case of positive multiplex results, a simplex analysis for confirmation and identification of mutations was performed. Four nanograms of cfDNA was pre-amplified (9 cycles) using $12.5 \mu \mathrm{L}$ of Q5 Hot Start High Fidelity Master Mix (New England Biolabs, Ipswich, MA, USA) and $0.7 \mu \mathrm{L}$ of $20 \mathrm{X}$ each mutation's assays (Thermo Fisher Scientific, Carlsbad, CA, USA) as previously described [8]. The following thermocycling PCR steps were used: $98^{\circ} \mathrm{C}, 3 \mathrm{~min} ; 9 \mathrm{cy}-$ cles: $98^{\circ} \mathrm{C}, 10 \mathrm{~s} ; 60^{\circ} \mathrm{C}, 3 \mathrm{~min} ; 72^{\circ} \mathrm{C}, 30 \mathrm{~s}$ and $72{ }^{\circ} \mathrm{C}, 2$ min. ddPCR analyses were performed following the manufacturer's recommendations using $2 \mu \mathrm{L}$ of preamplified cfDNA. The total copy number for each sample was systematically between 200 and 2000 copies/ $\mu \mathrm{L}$ per reaction. Negative control wells with no DNA were included in every run.

Background noise is the minimum concentration of the mutant allele that can be differentiated from a negative control. To assess the background noise of our method, the allele burden was measured in $11 \mathrm{cfDNA}$ (for multiplex analysis) and 11 pre-amplified cfDNA extracted from healthy control EDTA plasma samples collected under the same conditions as the patient samples. In this study regarding the theoretical sample's copy number and the background noise of each assay, a positive threshold of $0.1 \%$ was used.

All data were analyzed using QuantaSoft software (Bio-Rad) and were manually reviewed to provide a precise interpretation of the data points. The variant allele fraction (VAF) was defined as the proportion of mutant DNA copies relative to the sum of mutant and wild-type DNA copies obtained by ddPCR. Samples were considered mutated if at least two independent ddPCR analyses found a VAF above the mutation threshold. ddPCR analyses were all performed blindly from clinical data.

\section{Statistical analysis}

The primary objective was to determine the risk of progression or death at the next follow-up visit (after 3 months) according to circulating ESR1 mutation detection. The secondary objectives were to evaluate the risk of PD according to cfDNA and CA-15.3 increases and to determine the correlation between these biomarkers with progression or death. A predictive logistic regression model was thus performed, by using 
circulating ESR1 mutation status assessed every 3 months during follow-up. Based on our previous data, we hypothesized that $30 \%$ of the patients would have an ESR1 mutation detectable at progression. The observation of 56 progression events was required to detect at least a 2.4 risk ratio (RR) of progression or death until the next follow-up visit in the case of circulating ESR1 mutation detection, assuming an alpha risk of $5 \%$ and $80 \%$ power. Comparisons between groups were made using the chi-squared test for categorical variables and the Wilcoxon-Mann-Whitney test for quantitative variables. Survival curves were estimated by the Kaplan-Meier method and compared using the log-rank test. We also reported the $p$ value associated with the Cox model by considering CA15.3 and cfDNA as continuous variables and assessing a possible monotone association between CA-15.3 (or cfDNA) and survival, without fixing a threshold. All statistical analyses were performed using $\mathrm{R}$ software version 3.0.1.

\section{Results}

Patient characteristics, clinical follow-up, and outcome A total of $104 \mathrm{HR}+\mathrm{MBC}$ patients were prospectively included between June 2015 and August 2017. One patient was not considered for the analysis due to the lack of available plasma. The baseline characteristics of the 103 remaining patients are summarized in Table 1 . Of note, $28(27.2 \%)$ were already being treated with an $\mathrm{AI}$ at the time of inclusion. All patients had distant metastases except one who had tumor relapse with a deep invasion of the axillary fossa and permeation nodes. The median follow-up from AI initiation was 25 months (range 392). During that time, 70 patients (68\%) experienced progression of the disease (PD), and 20 patients (19\%) died. The median PFS and OS were 20.6 months and not achieved, respectively. PD was due to distant progression in $64 / 70$ patients (91\%) and to cutaneous progression with permeation nodes in $6 / 70$ patients.

\section{Biomarker analyses}

A total of 596 blood samples were analyzed during the study period corresponding to a median of 6 consecutive samples (range 2-9) per patient. Circulating ESR1 mutations were detectable at baseline in 4 patients: 1 already under AI treatment and 3 who initiated AI treatment at inclusion.

\section{Biomarker variations and correlation with progression Circulating ESR1 mutations}

Among the 70 patients who experienced PD during follow-up, circulating ESR1 mutations were detectable in 22 patients (31.4\%), including 18/22 (82\%) before PD and $4 / 22(18 \%)$ at the time of progression (Fig. 1). ESR1 mutation detection rate was not different between patients with de novo metastatic disease (11/53) and patients who relapsed after adjuvant treatment (11/50). Among the 22 patients with ESR1 mutations detected at or before PD, 15 patients (68\%) had a single mutation detected, while 7 patients had a polyclonal mutation. Among the 7 mutations tested, the D538G was the most frequent mutation, detected in $12 / 22$ patients (55\%). The detection rates for the other mutations were Y537S (10/ $22,45 \%), \mathrm{Y} 537 \mathrm{~N}(7 / 22,32 \%), \mathrm{E} 380 \mathrm{Q}$ and Y537C $(3 / 22$, 14\%), S463P (1/22, 5\%), and L536R (0/22). ESR1 mutation detection was significantly associated with $\mathrm{PD}$ $(p<0.001$, chi-square test), without detectable mutations at any time in patients without PD. Overall, the median time from ESR1 mutation detection to progression was 91 days [0-282] (Table 2).

\section{ESR1 mutation detection before progression}

Among patients with the emergence of circulating ESR1 mutations before PD $(n=18)$, the median delay was 110 days (range 50-282) from circulating ESR1 mutation detection to PD. The mutation was detected in every interval sample until progression in $15 / 18$ of the patients (83\%). Among the 3 patients with ESR1 mutation at the initiation of AI treatment (baseline), one had a clearance of the mutation from month 3 to PD observed at month 9. Another patient had a continuous increase of the ESR1 mutation value every 3 months until PD at month 6 . Finally, the third patient had a decrease of the mutation level detected between baseline and month 3, while PD occurred at month 3 . The presence of a circulating ESR1 mutation was significantly associated with the risk of PD at the next follow-up with a RR of 4.9 [3.0-8.0] at 3 months and 3.3 [2.4-4.5] at 6 months and an overall RR of 1.9 [1.7-2.0] compared to patients without ESR1 mutation detection $(p<0.001$ in each case, Table 3$)$.

\section{CA-15.3 increase during follow-up}

At baseline, median CA-15.3 value was $41 \mathrm{kUI} / \mathrm{L} \mathrm{[6-}$ 2454], and 61 patients (59\%) had a supranormal CA-15.3 value (> $30 \mathrm{kUI} / \mathrm{L})$. During follow-up, a CA-15.3 increase at a threshold of $25 \%$ was observed in $47 / 70(67 \%)$ patients with PD, including 27 (57\%) at the time of PD and 20 (43\%) before (Fig. 1). Among patients without PD, a $25 \%$ CA-15.3 increase was observed in 2 patients $(6 \%)$ (Table 2). A CA-15.3 increase at a threshold of $100 \%$ was observed in 23 (33\%) patients with PD, including 21 (84\%) at the time of PD and $2(16 \%)$ before (Fig. 1). Among the patients without PD, a $100 \%$ CA-15.3 increase was observed in 2 patients (6\%) (Table 2). Both CA-15.3 increases of $25 \%$ and $100 \%$ were correlated with PD $(p<0.001$, chi-square test) (Table 2$)$, with a median delay of 0 days from CA-15.3 increase to PD for both thresholds. 
Table 1 Baseline patient and disease characteristics

\begin{tabular}{|c|c|c|}
\hline Median age at inclusion (years) & 66 & [39-85] \\
\hline \multicolumn{3}{|l|}{ Performance status } \\
\hline 0 & 39 & $37.9 \%$ \\
\hline 1 & 46 & $44.7 \%$ \\
\hline 2 & 11 & $10.7 \%$ \\
\hline 3 & 2 & $1.9 \%$ \\
\hline NA & 3 & $2.9 \%$ \\
\hline BMI $\left(\mathrm{kg} / \mathrm{m}^{2}\right)$ & 27 & {$[18.3-56.5]$} \\
\hline \multicolumn{3}{|l|}{ HER2 status } \\
\hline Positive & 9 & $8.7 \%$ \\
\hline Negative & 90 & $87.4 \%$ \\
\hline NA & 4 & $3.9 \%$ \\
\hline \multicolumn{3}{|l|}{ Disease presentation at metastatic setting* } \\
\hline De novo & 53 & $51.4 \%$ \\
\hline Relapsed & 50 & $48.6 \%$ \\
\hline \multicolumn{3}{|l|}{ Adjuvant treatment } \\
\hline Chemotherapy & 37 & $74.0 \%$ \\
\hline Hormonotherapy & 44 & $88.0 \%$ \\
\hline Tamoxifen & 34 & $77.3 \%$ \\
\hline Al & 25 & $56.8 \%$ \\
\hline Median delay from end of adjuvant treatment to metastatic diagnostic (months) & 57.5 & [37-107] \\
\hline \multicolumn{3}{|l|}{ Metastatic treatment before Al introduction } \\
\hline \multicolumn{3}{|l|}{ Chemotherapy } \\
\hline Yes & 26 & $25.2 \%$ \\
\hline No & 77 & $74.8 \%$ \\
\hline \multicolumn{3}{|l|}{ Endocrine therapy except Al } \\
\hline Yes & 13 & $12.6 \%$ \\
\hline No & 90 & $87.4 \%$ \\
\hline \multicolumn{3}{|l|}{ Al status at inclusion } \\
\hline Initiation at inclusion & 75 & $72.8 \%$ \\
\hline Already started without progression & 28 & $27.2 \%$ \\
\hline Median delay since Al introduction (months) & 9.9 & {$[6.2-63.8]$} \\
\hline Median follow-up (months) & 25.3 & [3-92] \\
\hline
\end{tabular}

Data are presented as no. (\%) unless indicated otherwise

*Presentation of advanced disease is defined as de novo (advanced at first presentation) or relapsed (relapsed after previous presentation with early-stage cancer)

When a CA-15.3 increase of $25 \%$ was detected before progression, a median delay of 91 days [14-543] was observed from CA-15.3 increase to PD. The presence of a CA-15.3 increase of $25 \%$ was significantly associated with the risk of PD at the next follow-up with a RR of progression of 5.9 [3.8-9.2] at 3 months and 3.4 [2.54.8 ] at 6 months and an overall RR of 2.0 [1.7-2.4] compared to patients without a CA-15.3 increase $(p<0.001$ in each case, Table 3). Of note, a CA-15.3 increase of $100 \%$ was not associated with a significant RR of progression (chi-square test), probably because of the low number of patients with this threshold $(n=4)$ (Table 3).

\section{cfDNA increase during follow-up}

A cfDNA increase of $25 \%$ was observed in 59 patients (84\%) with PD, including 45 (76\%) before and 14 (34\%) at the time of progression (Fig. 1). Among patients without PD, a $25 \%$ cfDNA increase was observed in 32 patients (96\%). A threshold of cfDNA increase at $100 \%$ was identified in $33(47 \%)$ patients with PD, including 20 (61\%) before and $13(39 \%)$ at progression (Fig. 1). Among patients without PD, a 100\% cfDNA increase was observed in 22 patients (67\%). cfDNA increases of 25 or $100 \%$ were not significantly correlated with progression (chi-square test, Table 2). Overall, the presence 


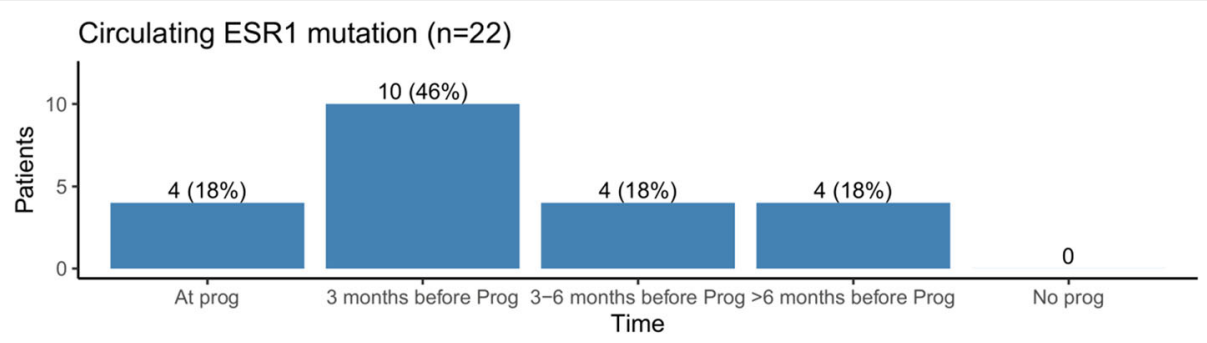

$25 \%$ CA 15.3 increase $(n=47)$

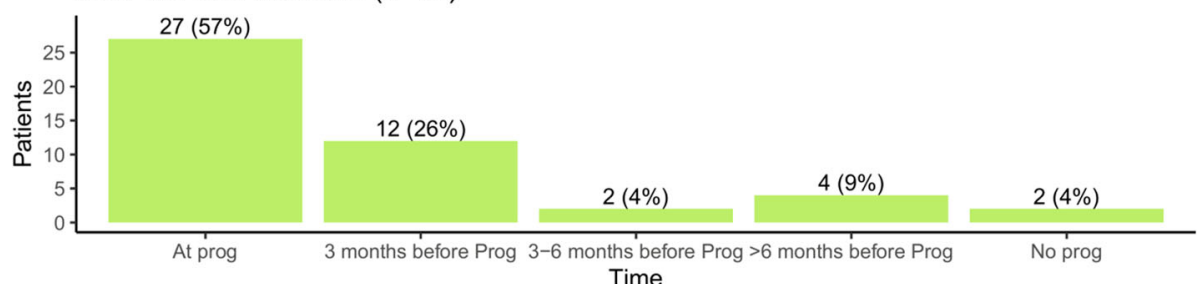

$100 \%$ CA 15.3 increase $(n=25)$

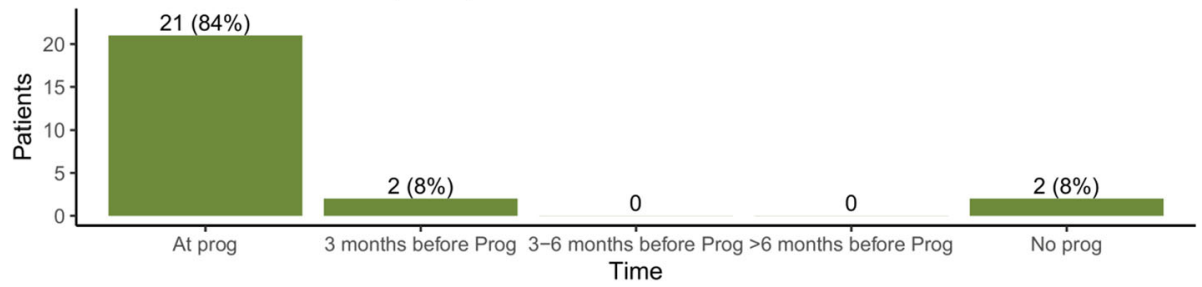

$25 \%$ cfDNA increase $(n=91)$

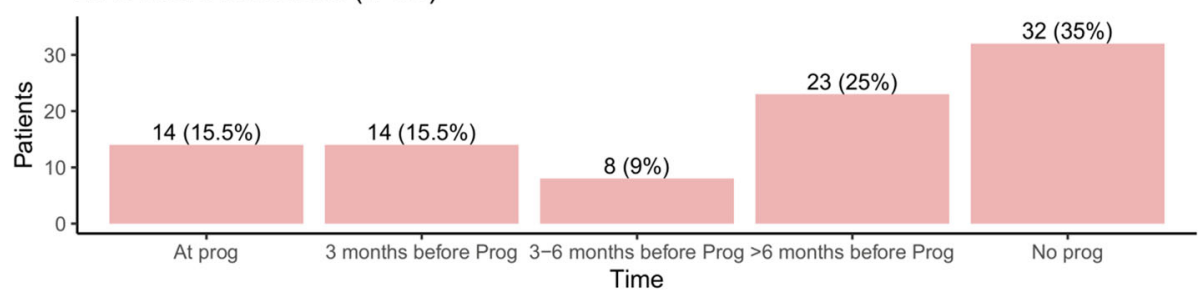

$100 \%$ cfDNA increase $(n=55)$

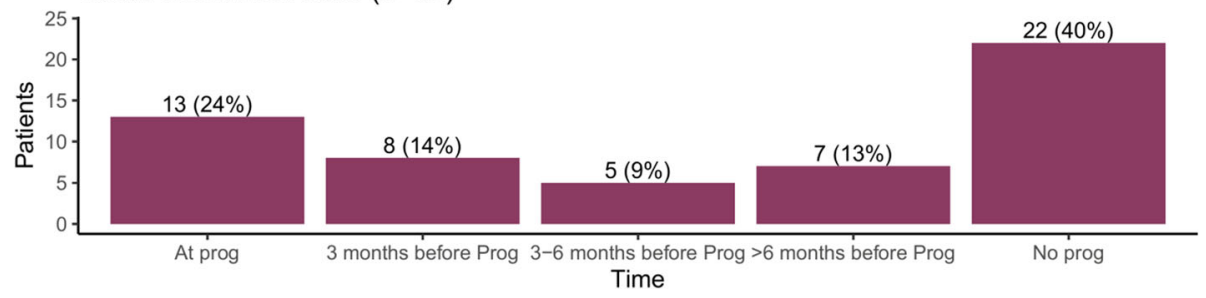

Fig. 1 Correlation between biomarker variations and time to progression. For each biomarker, the number of patients with a biomarker event (biomarker increase or mutation emergence) according to the time of progression (PD) is reported

of an increase in cfDNA using both thresholds was not associated with the risk of early PD (Table 3 ).

\section{Prognostic value of biomarkers at progression on Al}

Among the 75 patients with the initiation of AI treatment at inclusion, the median CA-15.3 and median cfDNA were not correlated with PFS or OS (see supplementary). At progression on $\mathrm{AI}$, and considering the low number of patients concerned, having a detectable circulating ESR1 mutation was not associated with a worse outcome (Fig. 2). Elevated CA-15.3 was related to a worse OS only when regarded as a continuous variable (Fig. 3 ), while cfDNA values at PD were related to a significantly worse outcome when used as a median value and continuous variable (Fig. 4).

\section{Discussion}

Our prospective study showed that the emergence of circulating ESR1 mutations is associated with a 4.9- and 
Table 2 Incidence of biomarker variations and correlations with progression

\begin{tabular}{|c|c|c|c|c|c|c|}
\hline \multirow[t]{2}{*}{ Marker } & \multicolumn{2}{|c|}{ All population } & \multirow{2}{*}{$\begin{array}{l}\text { Progression } \\
n=70\end{array}$} & \multirow{2}{*}{$\begin{array}{l}\text { No progression } \\
n=33\end{array}$} & \multirow[b]{2}{*}{$p$} & \multirow[b]{2}{*}{ Median delay (days) } \\
\hline & $n=103$ & $\%$ & & & & \\
\hline \multicolumn{7}{|l|}{ ESR1 } \\
\hline Mutated & 22 & $21 \%$ & $22(31 \%)$ & $0(0 \%)$ & $<0.001$ & $91[0-282]$ \\
\hline Non mutated & 81 & $79 \%$ & $48(69 \%)$ & $33(100 \%)$ & & \\
\hline \multicolumn{7}{|c|}{$>25 \%$ CA-15.3 increase } \\
\hline Yes & 47 & $46 \%$ & $45(64 \%)$ & $2(6 \%)$ & $<0.001$ & $0[0-543]$ \\
\hline No & 56 & $54 \%$ & $25(36 \%)$ & $31(94 \%)$ & & \\
\hline \multicolumn{7}{|c|}{$>100 \%$ CA-15.3 increase } \\
\hline Yes & 25 & $24 \%$ & $23(33 \%)$ & $2(6 \%)$ & 0.003 & 0 [0-91] \\
\hline No & 78 & $76 \%$ & 47 (67\%) & $31(94 \%)$ & & \\
\hline \multicolumn{7}{|c|}{$>25 \%$ DNA increase } \\
\hline Yes & 91 & $88 \%$ & 59 (84\%) & $32(97 \%)$ & 0.1 & $182[0-635]$ \\
\hline No & 12 & $12 \%$ & $11(16 \%)$ & $1(3 \%)$ & & \\
\hline \multicolumn{7}{|c|}{$>100 \%$ DNA increase } \\
\hline Yes & 55 & $53 \%$ & $33(42 \%)$ & $22(67 \%)$ & 0.1 & $92[0-474]$ \\
\hline No & 48 & $47 \%$ & 37 (58\%) & $11(33 \%)$ & & \\
\hline
\end{tabular}

$p$ values were determined using a chi-square test

3.3-fold increase in the risk of PD at 3 and 6 months, respectively, in comparison to patients without ESR1 mutations during AI treatment in $\mathrm{HR}+\mathrm{MBC}$. While CA15.3 was also significantly associated with an increased risk of PD, with a 5.9- and 3.4-fold increase at 3 and 6 months, respectively, our findings support that ESR1 monitoring is a better predictor than CA-15.3 in that setting. Indeed, we observed that circulating ESR1 mutations occurred in almost $75 \%$ of patients before clinical progression, in contrast to the CA-15.3 increase, which occurred in $57 \%$ of patients concomitantly with PD. To our knowledge, these results have never been reported so far and ESR1 tracking appears to date as the most clinically relevant marker for AI monitoring in $\mathrm{HR}+\mathrm{MBC}$.

As previously reported, circulating ESR1 mutations were found in $31.4 \%$ of patients at progression, with a $82 \%$ detection rate before PD $[8,14]$. The most frequent

Table 3 Risk ratio (RR) of progression according to each biomarker

\begin{tabular}{|c|c|c|c|c|c|}
\hline \multirow[t]{2}{*}{ Marker } & \multicolumn{3}{|l|}{ Progression } & \multirow{2}{*}{$\begin{array}{l}\text { No } \\
\text { progression }\end{array}$} & \multirow[t]{2}{*}{ Total } \\
\hline & $\leq 3$ months & $\leq 6$ months & Anytime after marker appearance & & \\
\hline \multicolumn{6}{|l|}{ ESR1 mutation } \\
\hline$n(\%)$ & $10(56 \%)$ & $14(78 \%)$ & $18(100 \%)$ & $0(0 \%)$ & $18(100 \%)$ \\
\hline RR (ref = non mut) & $4.9[3.0-8.0]$ & $3.3[2.4-4.5]$ & $1.9[1.7-2.0]$ & - & \\
\hline \multicolumn{6}{|l|}{$>25 \%$ CA-15.3 increase } \\
\hline$n(\%)$ & $12(60 \%)$ & $14(70 \%)$ & $18(90 \%)$ & $2(10 \%)$ & $20(100 \%)$ \\
\hline RR (ref = no increase) & $5.9[3.8-9.2]$ & $3.4[2.5-4.8]$ & $2.0[1.7-2.4]$ & - & \\
\hline \multicolumn{6}{|l|}{$>100 \%$ CA-15.3 increase } \\
\hline$n(\%)$ & $2(50 \%)$ & $2(50 \%)$ & $2(50 \%)$ & $2(50 \%)$ & $4(100 \%)$ \\
\hline RR (ref = no increase) & $4.0[1.5-11.0]$ & $2.2[0.8-5.8]$ & $1.1[0.4-2.8]$ & - & \\
\hline \multicolumn{6}{|l|}{$>25 \%$ DNA increase } \\
\hline$n(\%)$ & $14(18 \%)$ & $22(29 \%)$ & $45(58 \%)$ & $32(42 \%)$ & $77(100 \%)$ \\
\hline RR (ref = no increase) & $1.6[0.8-3.3]$ & $1.1[0.7-1.7]$ & $0.9[0.7-1.2]$ & - & \\
\hline \multicolumn{6}{|l|}{$>100 \%$ DNA increase } \\
\hline$n(\%)$ & $8(19 \%)$ & $13(31 \%)$ & $20(48 \%)$ & $22(52 \%)$ & $42(100 \%)$ \\
\hline RR (ref = no increase) & $1.4[0.7-2.7]$ & $1.1[0.6-1.7]$ & $0.8[0.6-1.2]$ & - & \\
\hline
\end{tabular}




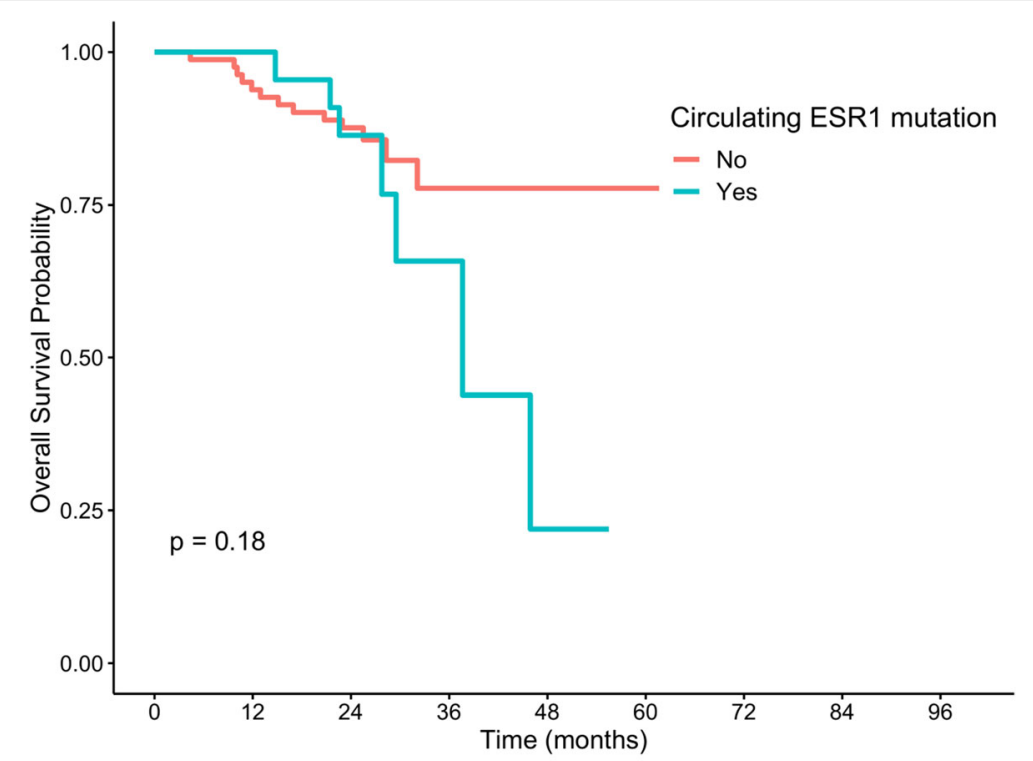

Number at risk

Yes. \begin{tabular}{ccccccccc}
81 & 76 & 67 & 10 & 7 & 4 & 3 & 2 & 0 \\
22 & 22 & 18 & 3 & 1 & 0 & 0 & 0 & 0 \\
\hline 0 & 12 & 24 & 36 & $\begin{array}{c}48 \\
\text { Time (months) }\end{array}$ & 72 & 84 & 96
\end{tabular}

Fig. 2 Overall survival according to ESR1 mutation status at progression disease. $p$ value was determined using a log-rank test

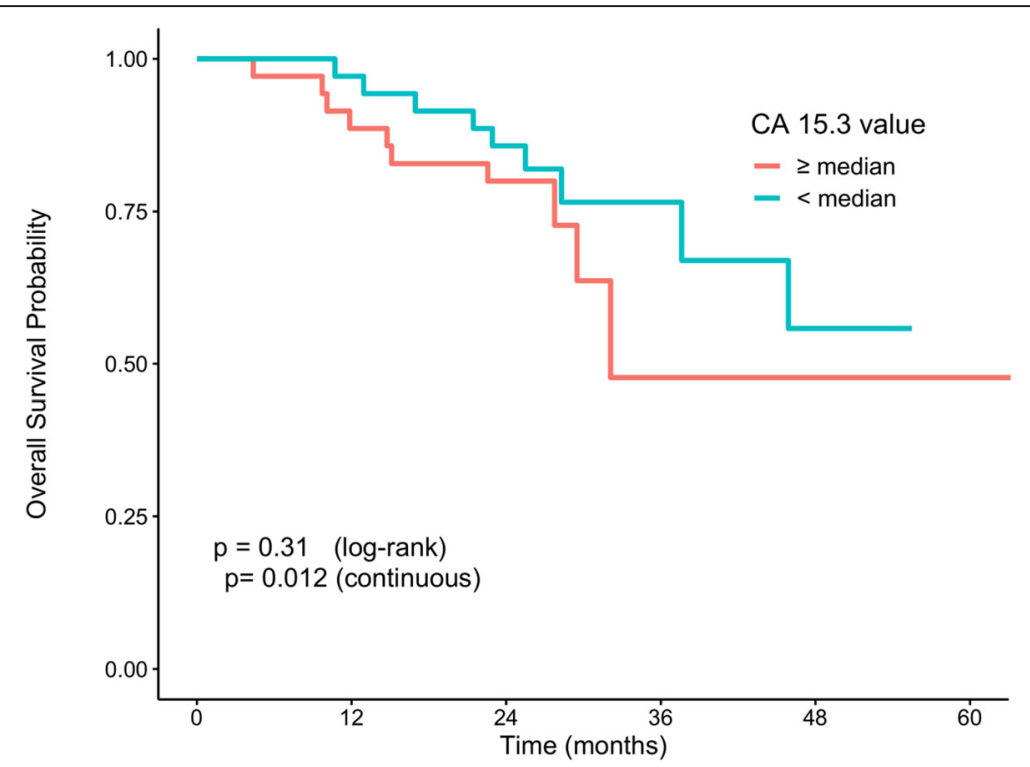

Number at risk

\begin{tabular}{|c|c|c|c|c|c|c|}
\hline$\geq$ median & 35 & 31 & 25 & 1 & 1 & 1 \\
\hline \multirow[t]{2}{*}{$<$ median } & 35 & 34 & 29 & 8 & 3 & 0 \\
\hline & 0 & 12 & $\begin{array}{l}24 \\
\text { Tin }\end{array}$ & $\begin{array}{l}36 \\
\text { s) }\end{array}$ & 48 & 60 \\
\hline
\end{tabular}

Fig. 3 Overall survival according to CA-15.3 level at progression disease. $p$ value was determined using a log-rank test or a Cox model 


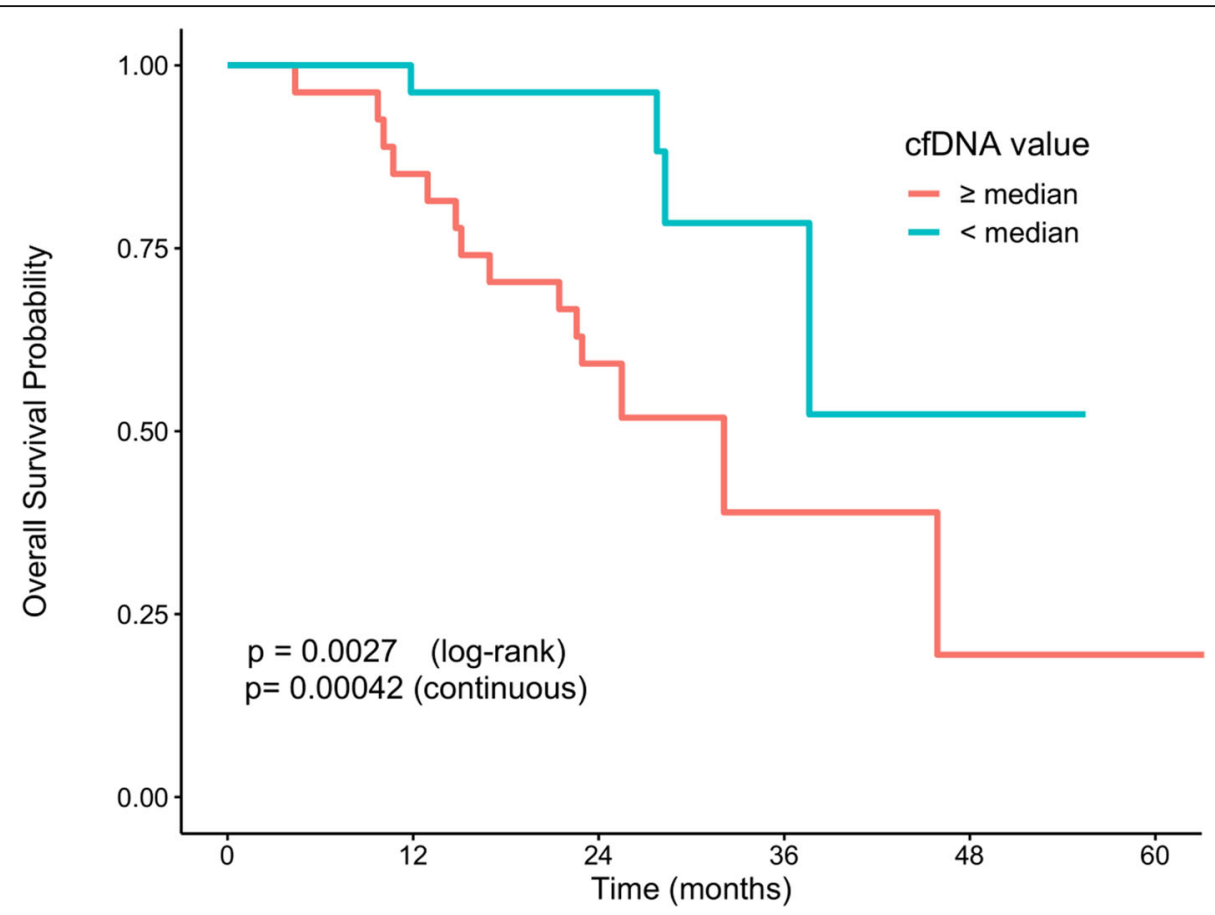

\begin{tabular}{|c|c|c|c|c|c|c|}
\hline \multicolumn{7}{|c|}{ Number at risk } \\
\hline$\geq$ median & 27 & 23 & 13 & 2 & 1 & 1 \\
\hline$<$ median & 27 & 26 & 25 & 3 & 2 & 0 \\
\hline & 0 & 12 & $\begin{array}{r}24 \\
\mathrm{Ti}\end{array}$ & $\begin{array}{r}36 \\
\text { is) }\end{array}$ & 48 & 60 \\
\hline
\end{tabular}

Fig. 4 Overall survival according to cfDNA level at progression disease. $p$ value was determined using a log-rank test or a Cox model

mutation identified was D538G, and polyclonal mutations were frequently observed (32\%), as previously reported $[8,17]$. To our knowledge, only one prospective study has evaluated the potential interest of ESR1 mutations in predicting clinical progression under AI treatment. In this study, Fribbens et al. followed 72 patients under first-line AI treatment and found an emergence of circulating ESR1 mutations in 22 of the $39(56 \%)$ patients with PD. As in our work, these circulating mutations were present before progression in 19/22 patients (86\%), with a median delay between the first circulating identification and PD of 6.7 months (range 3.7-NA). Our results are similar with $82 \%$ of circulating ESR1 mutations occurring before progression, with a slightly shorter median delay of 3.7 months [1.7-9.4]. Moreover, we have shown that all patients with detectable circulating ESR1 mutations progressed. In contrast, Fribbens et al. reported that $5 / 33(15 \%)$ of the patients who did not progress had a detectable ESR1 mutation without details regarding their clinical follow-up. To our knowledge, our study was the first to specifically quantify the risk of PD when ESR1 mutations occurred, with 4.9-fold and 3.3-fold increases at 3 and 6 months of follow-up, respectively. Interestingly, the ongoing phase III randomized trial PADA-1 (NCT03079011) is evaluating the potential value of early treatment modification in the case of emergent ESR1 mutation determined by ddPCR. Of note, ESR1 detection by ddPCR in daily practice could be easily implemented in a molecular laboratory since detection kits are commercialized. The main issue is the delay limited to few hours between sample collection and process when using EDTA tubes. But the use of PAXgene or Streck tubes allows cfDNA preservation at room temperature up to 7 days between sample collection and first centrifugation [18].

When considering CA-15.3, the results were not different regarding the $25 \%$ or $100 \%$ increase thresholds. We also found that there was a significant association between CA-15.3 increase and PD, with a majority of cases (57\%) occurring concomitantly with progression. Until now, CA15.3 remains the most frequent marker used in $\mathrm{HR}+\mathrm{MBC}$. Indeed, in previous studies focusing on the usefulness of CA-15.3 or carcinoembryonic antigen (CEA) to predict outcome, CA-15.3 is considered 
the best single biomarker in that setting [19-22]. The interest of biomarker combination including namely CA15.3 and CEA has been reported [23], even if the data remains conflicting with other work suggesting no increase of sensitivity and a decrease of the positive predictive value when considering a combination instead of CA-15.3 alone [19]. In our study, and in contrast to cfDNA assessment, CA 15.3 elevation was highly correlated to clinical evolution, and only 2 patients had a CA 15.3 increase $>25 \%$ without PD in the next months. However, it is noteworthy that since our design was based on a current follow-up of patients every 12 weeks, we have specifically planned the serum CA15.3 marker collection on the same schedule. Considering that 1month sampling interval of CA15.3 is commonly used in the scientific literature pursuing the same aim in the same setting, the sampling interval of 12 weeks for serum CA15.3 maybe represents a limitation of this work [23-25].

Regarding cfDNA, an increase (either using $25 \%$ or $100 \%$ threshold) was not correlated with progression. Moreover, most of the patients without progression had previously experienced a cfDNA increase. Even if we found that elevated cfDNA at progression was associated with poor prognosis, the lack of a correlation between cfDNA variation and clinical progression makes this biomarker unsuitable for daily practice. A high level of cfDNA has been previously related to OS at progression in $\mathrm{MBC}$ patients $[4,8,26,27]$, but to our knowledge, this is the first study that investigated the potential use of cfDNA to predict progression. Even if the total amount of cfDNA is correlated with tumor stage, many mechanisms other than tumor progression may lead to an increase in cfDNA, such as necrosis, autophagy, and hypoxia [28], which may explain the lack of correlation between cfDNA variations and $P D$. Recently, the results of a study comparing the correlation between CA-15.3, cfDNA, CTCs, and alkaline phosphatase values in $194 \mathrm{MBC}$ patients receiving various treatments, or not, were reported. The authors observed that cfDNA and CTCs were correlated with overall survival (HR 1.2 for both biomarkers), while cfDNA was the only biomarker correlated with progression-free survival. They concluded that a single cfDNA analysis could be an interesting biomarker for treatment evaluation in $\mathrm{MBC}$ patients. Their results are not comparable to ours since the population included was different, and since they considered an absolute value for each biomarker rather than a variation. Nevertheless, the poor AUC (0.593) that they observed when using cfDNA to discriminate patients who have responded or not makes this biomarker hard to use in daily practice for treatment adaptation [29].
This study has several limitations. First, 28/103 patients $(27 \%)$ were already under AI treatment without progression at inclusion in this study, and we cannot exclude that a biomarker variation occurred in the first months of AI exposure. Of note, only one patient out of these 28 had a circulating ESR1 mutation detected at inclusion. This mutation remained detectable in every 3month sample until PD 9 months after inclusion. Second, this study was conducted before the combination of cdk4/6 inhibitors and AI was established as a first-line therapy for HR+MBC. Thus, our results may not be applicable to patients with $\mathrm{AI}+\mathrm{cdk} 4 / 6$ inhibitors and dedicated studies are warranted in this population. On the other hand, recent results from the PALOMA-3 study revealed that ESR1 mutation was still an important mechanism of endocrine therapy resistance under treatment with cdk4/6 inhibitors, with a peculiar selection of the ESR1 Y537S mutation enrichment when patients are exposed to fulvestrant + palbociclib [30]. Third, in addition to circulating ESR1 mutations that are associated with resistance to $\mathrm{AI}$, other genomic alterations can be used to determine the amount of circulating ctDNA, such as PIK3CA mutations [17]. Due to the limited availability of plasma samples, we were unable to explore circulating molecular alterations other than ESR1 mutations. Besides the use of ctDNA in the detection of PD in advanced breast cancer, ctDNA has also been investigated in early breast cancer to predict relapse after the end of adjuvant treatment. Garcia-Murillas et al. recently reported a lead time between ctDNA detection and relapse of 10.7 months (95\% CI [8.1-19.1]) using a personalized ddPCR assay, and Coombes et al. reported a comparable lead time of 8.9 months (range 0.5-24) using NGS [31, 32]. Thus, it seems that ctDNA detection in the early setting may provide a longer lead time before relapse than for prediction of PD in the metastatic setting. Due to the limited data, no definitive conclusions can be drawn, but we may hypothesize that the higher tumor burden in the metastatic setting may lead to a shorter lead time between ctDNA detection and clinical progression.

\section{Conclusions}

The present prospective study led to the quantification of the risk of early PD when circulating ESR1 mutations emerge under $\mathrm{AI}$ treatment in $\mathrm{HR}+\mathrm{MBC}$, with an increase in the risk of progression of 4.9-folds at 3 months and 3.3-folds at 6 months compared to patients without ESR1 mutations. We also highlighted that ESR1 tracking was more relevant than the CA-15.3 increase that occurred in the majority of cases concomitantly with PD. Taken together, these results prompt the evaluation of a novel strategy of treatment based on circulating ESR1 detection. 


\section{Supplementary information}

Supplementary information accompanies this paper at https://doi.org/10. 1186/s13058-020-01290-x.

Additional file 1. Provides the following survival curves: PFS according to cfDNA level at baseline. OS according to cfDNA level at baseline. PFS according to CA-15.3 level at baseline. OS according to CA-15.3 level at baseline.

\section{Abbreviations}

Al: Aromatase inhibitor; CEA: Carcinoembryonic antigen; cfDNA: Cell-free DNA; CTC: Circulating tumor cell; ddPCR: Digital droplet polymerase chain reaction; ESR1: Estrogen receptor gene; HR+: Hormone receptor positive; MBC: Metastatic breast cancer; NGS: Next-generation sequencing; OS: Overall survival; PD: Progressive disease; PFS: Progression-free survival; TPA: Tissue polypeptide antigen; VAF: Variant allele fraction

\section{Authors' contributions}

Design: FC, AP, MB, DS, FDF. Supervision: FC, AP, LB, DR, NSV, FDF. Collection of clinical data: FC, CL, GE, LA, CG, CA, MF, LB, SG. Collection and preparation of biological samples: AP, CC, LB, SG, DR. Experiments: LB, AP, CC, SL, NSV. Data analysis: FC, AP, LB, JL, NSV, FDF. Preparation of the manuscript, table, and figures (all originals): FC, JL, FDF. The authors read and approved the final manuscript.

\section{Funding}

This study was supported by Astra Zeneca and by La Ligue Contre le Cancer de Haute-Normandie. There was no role of the sponsors in the design, collection, analysis, interpretation of data, and writing of the manuscript.

\section{Availability of data and materials}

The datasets used and/or analyzed during the current study are available from the corresponding author on reasonable request.

\section{Ethics approval and consent to participate}

This study was approved by the French National Drug Safety Agency (ANSM, 10 April 2015) and by an external ethics committee (Comité de protection des personnes Nord oust I, 26 March 2015). All patients provided informed consent before participation.

\section{Consent for publication}

Not applicable

\section{Competing interests}

The authors declare that they have no competing interests.

\section{Author details}

'Department of Medical Oncology, Centre Henri Becquerel, Rouen, France. ${ }^{2}$ Normandie Univ, UNIROUEN, Inserm U1245, IRON group, Rouen University Hospital, Normandy Centre for Genomic and Personalized Medicine, Rouen, France. '3 Department of Biopathology, Centre Henri Becquerel, Rouen, France. ${ }^{4}$ Clinical Research Unit, Centre Henri Becquerel, Rouen, France. ${ }^{5}$ Institut Normand du Sein, Centre François Baclesse, Caen, France. ${ }^{6}$ Department of Clinical Research and Innovation, Rouen University Hospital, Rouen, France.

Received: 14 January 2020 Accepted: 6 May 2020

Published online: 28 May 2020

\section{References}

1. Harris L, Fritsche $H$, Mennel R, Norton L, Ravdin P, Taube S, et al. American Society of Clinical Oncology 2007 update of recommendations for the use of tumor markers in breast cancer. J Clin Oncol Off J Am Soc Clin Oncol. 2007:25:5287-312

2. Bidard F-C, Peeters DJ, Fehm T, Nolé F, Gisbert-Criado R, Mavroudis D, et al. Clinical validity of circulating tumour cells in patients with metastatic breast cancer: a pooled analysis of individual patient data. Lancet Oncol. 2014;15: 406-14.

3. Bettegowda C, Sausen M, Leary RJ, Kinde I, Wang Y, Agrawal N, et al. Detection of circulating tumor DNA in early- and late-stage human malignancies. Sci Transl Med. 2014;6:224ra24.
4. Cheng J, Holland-Letz T, Wallwiener M, Surowy H, Cuk K, Schott S, et al. Circulating free DNA integrity and concentration as independent prognostic markers in metastatic breast cancer. Breast Cancer Res Treat. 2018;169:69-82.

5. Finn RS, Martin M, Rugo HS, Jones S, Im S-A, Gelmon K, et al. Palbociclib and letrozole in advanced breast cancer. N Engl J Med. 2016:375:1925-36.

6. Chandarlapaty S, Chen D, He W, Sung P, Samoila A, You D, et al. Prevalence of ESR1 mutations in cell-free DNA and outcomes in metastatic breast cancer: a secondary analysis of the BOLERO-2 clinical trial. JAMA Oncol. 2016;2:1310-5.

7. Gyanchandani R, Kota KJ, Jonnalagadda AR, Minteer T, Knapick BA, Oesterreich S, et al. Detection of ESR1 mutations in circulating cell-free DNA from patients with metastatic breast cancer treated with palbociclib and letrozole. Oncotarget. 2017:8:66901-11.

8. Clatot F, Perdrix A, Augusto L, Beaussire L, Delacour J, Calbrix C, et al. Kinetics, prognostic and predictive values of ESR1 circulating mutations in metastatic breast cancer patients progressing on aromatase inhibitor. Oncotarget. 2016;7:74448-59.

9. Chu D, Paoletti C, Gersch C, VanDenBerg DA, Zabransky DJ, Cochran RL, et al. ESR1 mutations in circulating plasma tumor DNA from metastatic breast cancer patients. Clin Cancer Res Off J Am Assoc Cancer Res. 2016;22: 993-9.

10. Sefrioui D, Perdrix A, Sarafan-Vasseur N, Dolfus C, Dujon A, Picquenot J-M, et al. Short report: monitoring ESR1 mutations by circulating tumor DNA in aromatase inhibitor resistant metastatic breast cancer. Int J Cancer J Int Cancer. 2015;137:2513-9.

11. Schiavon G, Hrebien S, Garcia-Murillas I, Cutts RJ, Pearson A, Tarazona N, et al. Analysis of ESR1 mutation in circulating tumor DNA demonstrates evolution during therapy for metastatic breast cancer. Sci Transl Med. 2015; 7:313ra182.

12. Guttery DS, Page K, Hills A, Woodley L, Marchese SD, Rghebi B, et al. Noninvasive detection of activating estrogen receptor 1 (ESR1) mutations in estrogen receptor-positive metastatic breast cancer. Clin Chem. 2015;61: 974-82.

13. Yanagawa T, Kagara N, Miyake T, Tanei T, Naoi Y, Shimoda M, et al. Detection of ESR1 mutations in plasma and tumors from metastatic breast cancer patients using next-generation sequencing. Breast Cancer Res Treat. 2017; 163:231-40.

14. Fribbens C, Garcia Murillas I, Beaney M, Hrebien S, O'Leary B, Kilburn L, et al. Tracking evolution of aromatase inhibitor resistance with circulating tumour DNA analysis in metastatic breast cancer. Ann Oncol Off J Eur Soc Med Oncol. 2018;29:145-53.

15. Dawson S-J, Tsui DWY, Murtaza M, Biggs H, Rueda OM, Chin S-F, et al. Analysis of circulating tumor DNA to monitor metastatic breast cancer. N Engl J Med. 2013:368:1199-209.

16. Eisenhauer EA, Therasse P, Bogaerts J, Schwartz LH, Sargent D, Ford R, et al. New response evaluation criteria in solid tumours: revised RECIST guideline (version 1.1). Eur J Cancer Oxf Engl 1990. 2009;45:228-47.

17. O'Leary B, Hrebien S, Morden JP, Beaney M, Fribbens C, Huang X, et al. Early circulating tumor DNA dynamics and clonal selection with palbociclib and fulvestrant for breast cancer. Nat Commun. 2018:9:896.

18. Gilson P. Enrichment and analysis of ctDNA. Recent Results Cancer Res Fortschritte Krebsforsch Progres Dans Rech Sur Cancer. 2020;215:181-211.

19. Martoni A, Zamagni C, Bellanova B, Zanichelli L, Vecchi F, Cacciari N, et al. CEA, MCA, CA 15.3 and CA 549 and their combinations in expressing and monitoring metastatic breast cancer: a prospective comparative study. Eur J Cancer Oxf Engl 1990. 1995;31A:1615-21.

20. Kurebayashi J, Yamamoto Y, Tanaka K, Kohno N, Kurosumi M, Moriya T, et al. Significance of serum carcinoembryonic antigen and CA 15-3 in monitoring advanced breast cancer patients treated with systemic therapy: a large-scale retrospective study. Breast Cancer Tokyo Jpn. 2003;10:38-44.

21. Tampellini M, Berruti A, Bitossi R, Gorzegno G, Alabiso I, Bottini A, et al. Prognostic significance of changes in CA 15-3 serum levels during chemotherapy in metastatic breast cancer patients. Breast Cancer Res Treat. 2006;98:241-8.

22. Laessig D, Nagel D, Heinemann V, Untch M, Kahlert S, Bauerfeind I, et al. Importance of CEA and CA 15-3 during disease progression in metastatic breast cancer patients. Anticancer Res. 2007:27:1963-8.

23. Sölétormos $G$, Nielsen D, Schiøler V, Mouridsen H, Dombernowsky P. Monitoring different stages of breast cancer using tumour markers CA 15-3, CEA and TPA. Eur J Cancer Oxf Engl 1990. 2004;40:481-6. 
24. Kurebayashi J, Nishimura R, Tanaka K, Kohno N, Kurosumi M, Moriya T, et al. Significance of serum tumor markers in monitoring advanced breast cancer patients treated with systemic therapy: a prospective study. Breast Cancer Tokyo Jpn. 2004;11:389-95.

25. Perrier A, Boelle P-Y, Chrétien Y, Gligorov J, Lotz J-P, Brault D, et al. An updated evaluation of serum sHER2, CA15.3, and CEA levels as biomarkers for the response of patients with metastatic breast cancer to trastuzumabbased therapies. PloS One. 2020;15:e0227356.

26. Shaw JA, Guttery DS, Hills A, Fernandez-Garcia D, Page K, Rosales BM, et al. Mutation analysis of cell-free DNA and single circulating tumor cells in metastatic breast cancer patients with high circulating tumor cell counts. Clin Cancer Res Off J Am Assoc Cancer Res. 2017;23:88-96.

27. Ye Z, Wang C, Wan S, Mu Z, Zhang Z, Abu-Khalaf MM, et al. Association of clinical outcomes in metastatic breast cancer patients with circulating tumour cell and circulating cell-free DNA. Eur J Cancer Oxf Engl 1990. 2019; 106:133-43.

28. Thierry AR, El Messaoudi S, Gahan PB, Anker P, Stroun M. Origins, structures, and functions of circulating DNA in oncology. Cancer Metastasis Rev. 2016; 35:347-76.

29. Fernandez-Garcia D, Hills A, Page K, Hastings RK, Toghill B, Goddard KS, et al. Plasma cell-free DNA (cfDNA) as a predictive and prognostic marker in patients with metastatic breast cancer. Breast Cancer Res BCR. 2019;21:149.

30. O'Leary B, Cutts RJ, Liu Y, Hrebien S, Huang X, Fenwick K, et al. The genetic landscape and clonal evolution of breast cancer resistance to palbociclib plus fulvestrant in the PALOMA-3 trial. Cancer Discov. 2018;8:1390-403.

31. Garcia-Murillas I, Chopra N, Comino-Méndez I, Beaney M, Tovey H, Cutts RJ, et al. Assessment of molecular relapse detection in early-stage breast cancer. JAMA Oncol. 2019:5(10):1473-8.

32. Coombes RC, Page K, Salari R, Hastings RK, Armstrong A, Ahmed S, et al. Personalized detection of circulating tumor DNA antedates breast cancer metastatic recurrence. Clin Cancer Res Off J Am Assoc Cancer Res. 2019;25: 4255-63.

\section{Publisher's Note}

Springer Nature remains neutral with regard to jurisdictional claims in published maps and institutional affiliations.

Ready to submit your research? Choose BMC and benefit from:

- fast, convenient online submission

- thorough peer review by experienced researchers in your field

- rapid publication on acceptance

- support for research data, including large and complex data types

- gold Open Access which fosters wider collaboration and increased citations

- maximum visibility for your research: over $100 \mathrm{M}$ website views per year

At $\mathrm{BMC}$, research is always in progress.

Learn more biomedcentral.com/submissions 